\title{
A DIOPHANTINE EQUATION OVER A FUNCTION FIELD
}

\author{
J. W. S. CASSELS
}

\section{Dedicated to Kurt Mahler on his 75th birthday}

(Received 9 January 1978)

Communicated by J. H. Coates

\begin{abstract}
Let $x_{0}, x_{1}, x_{2}, x_{3}$ be polynomials in a variable $t$ and with coefficients in a field $k$ of characteristic 0 . If $x_{0}^{2}+x_{1}^{2}=t\left(x_{2}^{2}-x_{3}^{2}\right)$ and $t\left(x_{0}^{2} x_{1}^{2}\right)=x_{2}^{2}+x_{3}^{2}$, then $x_{0}=x_{1}=x_{2}=x_{3}=0$. This partially answers a question of Pjatetskii-Šapiro and Šafarevič about the $K 3$-surface $x_{0}^{4}+x_{3}^{4}=x_{1}^{4}+x_{2}^{4}$. The proof uses a technique of M. R. Christie.
\end{abstract}

Subject classification (Amer. Math. Soc. (MOS) 1970): 14 J 25

\section{Introduction}

THEOREM 1. Let $k$ be a field of characteristic 0 and let $t$ be transcendental over $k$. Then there are no $k(t)$-rational points $\left(x_{0}, x_{1}, x_{2}, x_{3}\right)$ on the curve

$$
\begin{aligned}
x_{0}^{2}+x_{1}^{2} & =t\left(x_{2}^{2}-x_{3}^{2}\right), \\
t\left(x_{0}^{2}-x_{1}^{2}\right) & =x_{2}^{2}+x_{3}^{2} .
\end{aligned}
$$

This partially answers a question raised by Pjatetskii-Šapiro and Šafarevič (1971). Dem'janenko (1977) claims to have proved Theorem 1 without the restriction on the characteristic of $k$, but his argument appears to be incomplete (see Section 5 below). I cannot decide whether Theorem 1 remains true in prime characteristic. Theorem 1 will be deduced from:

THEOREM 2. Let $k, t$ be as in the enunciation of Theorem 1 . If $\xi, \eta \in k(t)$ satisfy

$$
\xi(\xi-1)\left(\xi-t^{4}\right)=\eta^{2}
$$


then

$$
\xi=0,1, t^{2} \text { or } t^{4} \text {. }
$$

Theorem 2 will be proved by a technique of M. R. Christie (1976). There is clearly no loss of generality in proving Theorems 1 and 2 under the additional assumption that $k$ is algebraically closed.

\section{Deduction of Theorem 1 from Theorem 2}

Put

$$
\begin{array}{ll}
a=x_{0}+i x_{1}, & b=x_{0}-i x_{1}, \\
c=x_{2}+x_{3}, & d=x_{2}-x_{3},
\end{array}
$$

so that (1.1) and (1.2) become

$$
\begin{gathered}
a b=t c d, \\
t\left(a^{2}+b^{2}\right)=c^{2}+d^{2} .
\end{gathered}
$$

Put

so that by (2.3) we have

$$
\alpha=a / c, \quad \beta=a / d
$$

$$
a: b: c: d=\alpha \beta: t: \beta: \alpha .
$$

Then (2.5) becomes

$$
\alpha^{2}+\beta^{2}=t\left(t^{2}+\alpha^{2} \beta^{2}\right)
$$

that is

$$
\left(\alpha^{2}-t^{3}\right)=\beta^{2}\left(t \alpha^{2}-1\right)
$$

Put

$$
\xi=t \alpha^{2}
$$

Then

where

$$
\xi(\xi-1)\left(\xi-t^{4}\right)=\eta^{2}
$$

$$
\eta=t \alpha \beta\left(t \alpha^{2}-1\right) .
$$

If now $\xi$ is given by (1.4), then there is clearly no $\alpha$ satisfying (2.9).

\section{Theorems of Christie and Hellegouarch}

We enunciate some results which we shall need later.

Let $K$ be any field of characteristic 0 , let $k$ be any algebraically closed field containing $K$ and let $t$ be transcendental over $k$. Suppose that

$$
u, v \in K[t],
$$

that

$$
u \neq 0, \quad v \neq 0,
$$


and that

$$
u / v \notin K
$$

Then

$$
\xi(\xi-u)(\xi-v)=\eta^{2}
$$

is an elliptic curve defined over $K(t)$ but not $k(t)$-equivalent to an elliptic curve defined over $k$. We denote by $G$ the group of points on (3.4) defined over $k(t)$, so $G$ is finitely generated by the function-field analogue of the Mordell-Weil Theorem.

For $d \in K^{*}$, we consider also the curve

$$
x(x-u)(x-v)=d y^{2}
$$

and denote the group of points defined over $K(t)$ by $H(d)$. We shall identify $H(d)$ with a subgroup of $G$ by putting $\xi=x, \eta=d^{\frac{1}{b}} y$.

Theorem 3 (Christie (1976), Proposition 2). Suppose that $u, v$ and $u-v$ all split into the product of linear factors in $K[t]$. Then there is a finite set $D \subset K^{*}$ such that the $H(d), d \in D$ generate a group of finite index in $G$.

Christie considers only the case when $k$ is the field of complex numbers and $K$ is algebraic over $\mathbf{Q}$ but his argument is clearly general. He writes $k$ for our $K$ and works with $a, b$ where $u=a+2 b, v=a-2 b$.

We need only the

COROllary. Suppose that $G$ is infinite. Then $H(d)$ is infinite for some $d \in K^{*}$.

We also require:

THEOREM 4 (Hellegouarch (1970), Théorème 8). Let $t$ be transcendental over the field $k$ of characteristic 0 and let $u \neq 0, v \neq 0$ be elements of $k[t]$ such that $u / v \notin k$. Then the group of points $(\xi, \eta)$ on (3.4) defined over $k(t)$ has no p-torsion, where $p$ is any prime other than 2 or 3.

COROLlary. Suppose that $G$ has p-torsion for some $p \neq 2$. Then there is a point $(\alpha, \beta)$ on (3.4) with $\alpha, \beta \in k(t)$ and

$$
3 \alpha^{4}-4(u+v) \alpha^{3}+6 u v \alpha^{2}+u^{2} v^{2}=0 .
$$

For, as Christie (1976) remarks (end of his Section 3), this is the condition that $(\alpha, \beta)$ have order 3 .

We also recall for convenience 
Lemma 1. Let $L$ be a field of characteristic $\neq 2$ and let $M=M(\sqrt{ } s)$ for $s \in L$. Suppose that there are infinitely many points defined over $M$ on the elliptic curve

$$
f(\xi)=\eta^{2},
$$

where $f(\xi) \in L[\xi]$ is a cubic polynomial. Then there are infinitely many points defined over $L$ either on (3.7) or on

$$
f(\xi)=s \eta^{2}
$$

LemMA 2. Let $e_{1}, e_{2}, e_{3}$ be distinct elements of a field $L$ of characteristic $\neq 2$ and let $G$ be the Mordell-Weil group of the elliptic curve

$$
\left(\xi-e_{1}\right)\left(\xi-e_{2}\right)\left(\xi-e_{3}\right)=\eta^{2} .
$$

For $j=1,2,3$ let $\varphi_{j}$ be the map from $G$ to $L^{*} /\left(L^{*}\right)^{2}$ defined by

$$
\varphi_{j}(\xi, \eta)= \begin{cases}\left(\xi-e_{j}\right)\left(L^{*}\right)^{2} & \text { if } \xi \neq e_{j} \\ \left(e_{l}-e_{j}\right)\left(e_{m}-e_{j}\right)\left(L^{*}\right)^{2} & \text { if } \xi=e_{j}, \text { where } l \neq m \neq j \neq l .\end{cases}
$$

Then $\varphi_{j}$ is a group homomorphism. Further,

$$
2 G=\bigcap_{j=1}^{3} \operatorname{Ker}\left(\varphi_{j}\right) .
$$

\section{Proof of Theorem 2}

The points on (1.4) with $\xi=0,1, t^{4}$ are of order 2 and those with $\xi=t^{2}$ are of order 4. It is routine to show using Lemma 2 that there is no further 2-torsion and an application of Theorem 4, Corollary shows that there is no further torsion. We shall suppose that there are infinitely many points on (1.4) defined over $k(t)$ and will ultimately arrive at a contradiction.

We first apply Lemma 1 with $s=t^{2}$ and $L=k(s)$. There will thus be infinitely many points defined over $k(s)$ on at least one of the curves

$$
\begin{aligned}
& \xi(\xi-1)\left(\xi-s^{2}\right)=\eta^{2}, \\
& \xi(\xi-1)\left(\xi-s^{2}\right)=s \eta^{2} .
\end{aligned}
$$

To (3.4) we apply the same argument. If it has infinitely many $k(s)$-points, then there are infinitely many $k(r)$-points on one of

$$
\begin{aligned}
& \xi(\xi-1)(\xi-r)=\eta^{2}, \\
& \xi(\xi-1)(\xi-r)=r \eta^{2},
\end{aligned}
$$

where $r=s^{2}=t^{4}$ is transcendental over $k$. 
The curve (4.4) reduces to (4.3) on taking $r^{-1} \xi, r^{-1} \eta, r^{-1}$ for $\xi, \eta, r$ respectively. Hence we need consider only (4.2) over $k(s)$ and (4.3) over $k(r)$. We suppose, as we may, that $k$ is algebraically closed.

The equation (4.3) requires no deep machinery. On considering possible common factors on the left-hand side we have

$$
\begin{gathered}
\xi=r^{\alpha} \lambda^{2}, \\
\xi-1=(r-1)^{\beta} \mu^{2}, \\
\xi-r=r^{\alpha}(r-1)^{\beta} \nu^{2},
\end{gathered}
$$

where $\lambda, \mu, \nu \in k(r)$ and $\alpha, \beta=0$ or 1 . Hence, by Lemma $2, G^{*} / 2 G^{*}$ has order at most $2^{2}$, where $G^{*}$ is the group of points defined over $k(r)$. Since all the points of order 2 are defined over $k(r)$, there can thus be no points of infinite order.

There remains (4.2). We put

$$
X=s \xi, \quad Y=s^{2} \eta
$$

and so have to consider

$$
X(X-s)\left(X-s^{3}\right)=Y^{2}
$$

over $k(s)$. On considering common factors of the factors on the left-hand side we have

$$
\begin{gathered}
X=s^{\alpha} \lambda^{2}, \\
X-s=s^{\beta}(s-1)^{\delta}(s+1)^{\varepsilon} \mu^{2}, \\
X-s^{3}=s^{\gamma}(s-1)^{\delta}(s+1)^{\varepsilon} \nu^{2},
\end{gathered}
$$

where $\lambda, \mu, \nu \in k(s)$ and $\alpha, \beta, \gamma, \delta, \varepsilon=0$ or 1 with $\alpha+\beta+\gamma \equiv 0(\bmod 2)$. On considering $(X, Y)+\left(X_{0}, Y_{0}\right)$ if necessary, where $Y_{0}=0$ and $X_{0}=0, s$ or $s^{3}$, we may suppose by Lemma 2 without loss of generality that

$$
\alpha=\beta=\gamma=0 .
$$

We now apply Theorem 3 Corollary with $K=\mathbf{Q}, s$ instead of $t$, and $u=s, v=s^{3}$. If there are infinitely many points on (4.9) over $k(s)$ then there is some $d \in \mathbf{Q}^{*}$ such that there are infinitely many points over $\mathbf{Q}(s)$ on

$$
x(x-s)\left(x-s^{3}\right)=d y^{2} .
$$

By (4.10)-(4.13) we need consider only the following four cases, in all of which

$$
l, m, n \in \mathbf{Q}^{*}, \quad l m n \in d\left(\mathbf{Q}^{*}\right)^{2}
$$


and

$$
\begin{aligned}
& U, V, W \in \mathbf{Q}(s): \\
& \left.\begin{array}{rl}
x & =I U^{2} \\
x-s & =m V^{2} \\
x-s^{3} & =n W^{2}
\end{array}\right\} \\
& \left.\begin{array}{rl}
x & =l U^{2} \\
x-s & =m\left(s^{2}-1\right) V^{2}, \\
x-s^{3} & =n\left(s^{2}-1\right) W^{2}
\end{array}\right\} \\
& \left.\begin{array}{rl}
x & =l U^{2}, \\
x-s & =m(s-1) V^{2}, \\
x-s^{3} & =n(s-1) W^{2} ;
\end{array}\right\} \\
& \left.\begin{array}{rl}
x & =l U^{2} \\
x-s & =m(s+1) V^{2} \\
x-s^{3} & =n(s+1) W^{2} .
\end{array}\right\}
\end{aligned}
$$

We consider these in turn.

Case I. We have

$$
l U^{2}-s=m V^{2} .
$$

On localizing at $s=0$, this is clearly seen to imply that

Similarly

$$
l / m \in\left(\mathbf{Q}^{*}\right)^{2} \text {. }
$$

and so by (4.15),

$$
l / n \in\left(\mathbf{Q}^{*}\right)^{2}
$$

$$
l, m, n \in d\left(Q^{*}\right)^{2} \text {. }
$$

Hence $(x, y)=2\left(x_{1}, y_{1}\right)$ by Lemma 2 , where $\left(x_{1}, y_{1}\right)$ is a point on (4.14) defined over $\mathbf{Q}(s)$. Since the group of points on (4.14) over $\mathbf{Q}(s)$ is finitely generated, if there are infinitely many such points, then there will be some of them not in Case I.

Case II. We have

$$
l U^{2}-s=m\left(s^{2}-1\right) V^{2}
$$

On localizing at $s=1$, we have

$$
l \in\left(\mathbf{Q}^{*}\right)^{2}
$$


and on localizing at $s=-1$ we have

$$
-l \in\left(Q^{*}\right)^{2} .
$$

Hence Case II cannot occur.

Case III. We have

$$
l U^{2}-s=m(s-1) V^{2} .
$$

On localizing at $s=1$, we have

$$
l \in\left(\mathbf{Q}^{*}\right)^{2}
$$

and on localizing at $s=0$ we have

$$
-l / m \in\left(\mathbf{Q}^{*}\right)^{2} .
$$

Similarly,

$$
l U^{2}-s^{3}=n(s-1) W^{2}
$$

and so

$$
-l / n \in\left(\mathbf{Q}^{*}\right)^{2}
$$

Hence, on absorbing elements of $\mathbf{Q}^{*}$ into $U, V, W$, we need consider only

$$
\begin{gathered}
U^{2}-s=(1-s) V^{2}, \\
U^{2}-s^{3}=(1-s) W^{2} .
\end{gathered}
$$

On specializing $s$ to 2 we have

$$
\begin{aligned}
& u^{2}-2 h^{2}=-v^{2} \\
& u^{2}-8 h^{2}=-w^{2}
\end{aligned}
$$

where $u, v, w, h \in \mathbf{Q}$ and are not all zero. By homogeneity we may suppose that $u, h$ are integers without common factor. Then (4.17) implies that $u$ is odd, whereas (4.18) implies that $u$ is even. The contradiction shows that Case III cannot occur.

Case $I V$. This reduces to Case III on changing the signs of $X$ and $s$.

This concludes the proof of Theorem 2.

Hellegouarch's proof of Theorem 4 is somewhat obscure and so we note that it is not really essential to our argument for the following two reasons.

(i) We could have used the analogue of the Nagell-Lutz Theorem for $k(t)$. This asserts that if $f(\xi)$ is a cubic with coefficients in $k[t]$ and top coefficients 1 , and if $\xi, \eta$ is a point of finite order defined over $k(t)$ on $y^{2}=f(\xi)$, then $\xi, \eta \in k[t]$ and either $\eta=0$ or $\eta^{2}$ divides the discriminant of $f$. This reduces the determination of 
the torsion on (1.3) to a rather tedious case-by-case discussion. (It is enough to look at the odd torsion on (4.2) and 4.9).)

(ii) So far as the proof of Theorem 1 is concerned, it would, in any case, be enough to have the weaker form of Theorem 2 which asserts that there are no points of infinite order on (1.3) and that the 2-torsion is given by (1.4). It then follows from Lemma 2 that $\xi$ is a square for all torsion points: so (2.9) cannot hold.

\section{Dem'janenko's argument}

By a process similar to that in our Section 2, Dem'janenko deduces Theorem 1 from the assertion that the points $(u, v, w)$ defined over $k(t)$ on

satisfy

$$
u^{4}-2\left(2 t^{4}-1\right) u^{2} w^{2}+w^{4}=v^{2}
$$

$$
u=0 \text { or } w=0 \text { or } u^{2}+w^{2}=0 .
$$

By homogeneity we may suppose that $u, v, w \in k[t]$. Dem'janenko then considers a point $(u, v, w)$ for which $u \neq 0, w \neq 0$ and

$$
\operatorname{deg} u+\operatorname{deg} w
$$

is minimal. By a descent argument he shows that then (5.2) holds. This does not, however, imply that (5.2) holds for every solution $u, v, w$. It does not seem to me that Dem'janenko's argument can be modified so as to give a proof. Since (5.1) is isogenous to (1.3) we have, however, shown that Dem'janenko's assertion is true in characteristic 0 .

Added IN Proof. Professor Swinnerton-Dyer has shown me a geometric proof of Theorem 1 which extends to some (but not all) finite characteristics.

\section{References}

M. R. Christie (1976), "Positive definite functions of two variables which are not the sum of three squares", J. Number Theory 8, 224-232.

V. A. Dem'janenko (1977), "An indeterminate equation" (Russian), Zap. Naučn, Sem. Leningrad. Otdel. Mat. Inst. Steklov (LOMI), 67, 163-166.

Y. Hellegouarch (1970), "Étude des points d'ordre fini des variétés abeliennes de dimension un définies sur un anneau principal", J. reine. angew. Math. 244, 20-36.

I. I. Pjatetskii-Šapiro and I. R. Šafarevič (1971), "Torelli’s theorem for K3 algebraic surfaces" (Russian), Izv. Akad. Nauk SSSR (ser. mat.) 35, 530-572, especially the last section.

Department of Pure Mathematics and Mathematical Statistics

16 Mill Lane

Cambridge CB2 1SB

United Kingdom 